\title{
NeuroImage
}

ELSEVIER

\section{Graded recall success: an event-related fMRI comparison of tip of the tongue and feeling of knowing}

\author{
Anat Maril, ${ }^{\mathrm{a}, *}$ Jon S. Simons, ${ }^{\mathrm{a}, \mathrm{b}}$ Josh J. Weaver, ${ }^{\mathrm{a}}$ and Daniel L. Schacter ${ }^{\mathrm{a}}$

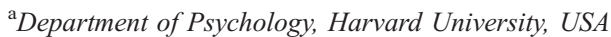 \\ ${ }^{\mathrm{b}}$ Institute of Cognitive Neuroscience, University College London, UK
}

Received 17 May 2004; revised 5 October 2004; accepted 25 October 2004

Available online 15 December 2004

\begin{abstract}
Insights into memory retrieval processes can be obtained by examining graded recall success, specifically, tip-of-the-tongue (TOT) and feelingof-knowing (FOK) states. TOT is defined as a recall failure accompanied by a strong feeling of imminent retrieval, and FOK as a recall failure accompanied by a feeling of future ability to recognize the item. The present fMRI study examined the brain regions associated with both intermediate retrieval states in a within-subject, within-memory system design. Subjects were presented with general knowledge questions and were instructed to respond to each with one of four options: Know, indicating successful retrieval of the answer; TOT; FOK; or Don't know, indicating retrieval failure. Different patterns of activation in several brain regions including prefrontal cortex were associated with TOT and FOK states. For example, TOT was associated with activation in anterior cingulate, right dorsal and inferior, and bilateral anterior, prefrontal cortex. TOT and FOK elicited similar levels of activation in parietal regions, both significantly greater than that associated with Know and Don't know responses. The results are interpreted in the light of theories of the role of prefrontal cortex in recall and cognitive conflict.
\end{abstract}

(c) 2004 Elsevier Inc. All rights reserved.

Keywords: Tongue; Feeling; Knowing

\section{Introduction}

The tip-of-the-tongue (TOT) state refers to a temporary inaccessibility of information that one is sure exists in long-term memory and is on the verge of recovering (Brown and McNeill, 1966; Schwartz, 2002). As such, the TOT represents an intermediate level of recall success in which the sought-after information is not recalled, but at the same time, it is distinctly different from a retrieval failure. Aside from people's subjective sense of imminent successful recall, behavioral data indicate that

* Corresponding author. Department of Neurology, Children's Hospital Boston, 300 Longwood Avenue, Pavilion 152, Boston, MA 02115, USA. Fax: +16174963122 .

E-mail address: anat.maril@childrens.harvard.edu (A. Maril).

Available online on ScienceDirect (www.sciencedirect.com). partial information about the target can often be retrieved, such as initial and last letter (Koriat and Leiblich, 1974), number of syllables (Rubin, 1975), syntactic properties (Miozzo and Caramazza, 1997; Vigliocco et al., 1999), and similar words that are related in sound or meaning (Cohen and Faulkner, 1986; Reason and Lucas, 1984).

In a recent event-related fMRI study of TOT (Maril et al., 2001), subjects were presented with general knowledge questions in the form of pairs of semantic cues (e.g., Chinatown + Director; Carmen + Composer) that converged on a target (e.g., Roman Polanski; Georges Bizet). Comparing TOT trials to successful (Know, K) and unsuccessful (Don't Know, DK) trials revealed significantly greater activation for TOT within anterior cingulate cortex (ACC), right dorsolateral prefrontal cortex (right DLPFC) and right inferior prefrontal cortex (RIFC). Activation in these regions was not modulated by $\mathrm{K}$ or DK responses; it remained close to baseline. These results were interpreted in light of what is known from behavioral studies of the TOT and from imaging and neuropsychological studies regarding the likely functions of the activated regions. Thus, partially recovered knowledge that may be related to the target or to similar words, such as first letter of the target item, similar names, or related semantic knowledge gives rise to a higher need for conflict management and postretrieval monitoring: each such retrieved item needs to be evaluated for accuracy and relevance, be used to stimulate further search, etc. These processes are thought to recruit the ACC-right DLPFC circuit (e.g., MacDonald et al., 2000) that serves to detect conflict (e.g., if a retrieved item is evaluated as hindering search for the target) and guide further retrieval attempts in search for the correct answer.

Though activation in these regions seems to be specifically related to the TOT in Maril et al.'s (2001) experiment, it is possible that the engagement of such areas might also characterize other recall states of a phenomenologically similar nature. Cognitive studies have explored another type of intermediate recall state, termed feeling-of-knowing (FOK). FOK refers to retrieval situations in which individuals fail to recall an item from memory, but at the same time are able to make an accurate prediction about their ability to recognize the item in a future test 
(Hart, 1965). Unlike TOT, during FOK people do not typically experience a feeling of imminent successful recall. Nevertheless, both recall states share the quality of being experienced as neither successful recall nor a complete failure of retrieval, but as 'intermediate' states. It is possible, then, that the apparent phenomenological similarity could be reflected in the involvement of similar cognitive processes, in which case, it would be expected that TOT-related regions would additionally be engaged in FOK situations.

A recent fMRI study compared FOK states to successful (K) and unsuccessful (DK) states in an episodic memory paradigm (Maril et al., 2003). Subjects encoded pairs of unrelated words, and when scanned were given the first member of the pair and asked to recall the second. They had to press a key for each of the three possible responses: $\mathrm{K}$, DK, or FOK. Using this paradigm, no region was observed that exhibited significantly greater activation for FOK trials than for $\mathrm{K}$ or DK trials, thus, contrasting sharply with the previously observed pattern for TOT. Rather, the pattern of neural activation in several left prefrontal regions along the inferior frontal gyrus, and parietal regions (Brodmann's area (BA) 7) tracked the phenomenological experience of the FOK intermediate state of retrieval. Successful recall trials were associated with the greatest activation in these regions, followed by an intermediate level of activation for FOK trials, which was in turn larger than that associated with failed recall trials. Thus, the FOK experience seemed to be associated with a particular pattern of neural activation; however, at least in these specific experimental circumstances, the pattern was distinctly different from that associated with TOT, as originally observed by Maril et al. (2001). It is of course problematic to compare patterns of activation across studies. In this case, comparison is especially difficult because the TOT and FOK experiences occurred during tasks that used different materials (general knowledge questions vs. paired associates, respectively) and even tapped different memory systems (semantic vs. episodic memory, respectively).

The purpose of the present study is to provide a more rigorous comparison of brain activation during TOT and FOK experiences: both TOT and FOK responses were examined in a withinmemory system, within-subject design using the same materials. In particular, we addressed the question of whether the pattern of activation in the "TOT specific" regions described by Maril et al. (2001) is indeed specific to TOT, or whether seemingly similar intermediate recall states, such as FOK, would elicit the same pattern when observed under the same experimental conditions. During scanning, subjects were presented with pairs of semantic cues that converge on a target in the same way described in Maril et al. (2001). They were given $4 \mathrm{~s}$ to indicate whether they knew the target name, did not know the target, experienced a TOT, or had FOK. An event-related analysis was then conducted, in which all trial types were examined relative to each other and to low-level fixation.

To the extent that TOT-related activation is indeed unique to TOT, and the pattern of activation previously associated with FOK is not limited to an episodic memory paradigm, we expected to replicate the TOT results (Maril et al., 2001), but to observe a different pattern of activation for FOK trials, perhaps similar to that previously observed (Maril et al., 2003). If, however, these two retrieval states are associated with similar processes, we would expect FOK trials to elicit similar levels of activation in TOT-related regions.

\section{Materials and methods}

Subjects

Participants were 15 right-handed, native speakers of English (7 men; ages 18-23 years), with normal or corrected-to-normal vision. Participants received $\$ 50$ for participation. A total of 22 subjects were scanned: data from five participants were excluded due to poor task performance, and data from two other subjects were excluded due to technical problems with scanner or peripheral equipment. Informed consent was obtained in a manner approved by the Human Studies Committee of the Massachusetts General Hospital.

\section{Stimuli and cognitive task}

Across two scans, 336 two-part general knowledge questions cued retrieval. Generally, stimuli from Maril et al. (2001) were used, with some items deleted (those that elicited a DK response from all the subjects in Maril et al., 2001). Each question was presented for $3 \mathrm{~s}$, followed by $1 \mathrm{~s}$ of visual fixation. Additional periods of baseline fixation, lasting between 2 and $10 \mathrm{~s}$, were interspersed between the experimental trials to optimize the efficiency of the design matrix (Dale, 1999). For experimental trials, subjects responded to each trial by pressing one of four response keys to indicate their retrieval outcome-successful retrieval ("Know"), unsuccessful retrieval not accompanied by a TOT or FOK ("Don't know"), unsuccessful retrieval accompanied by a TOT ("TOT') or unsuccessful retrieval accompanied by a FOK ("FOK"). Prior to the experiment, detailed instructions were provided to clarify what constitutes a TOT state, FOK state, and the difference between them. To verify, albeit indirectly, the responses that subjects provided during scanning, all questions were subsequently presented again outside of the scanner approximately $24 \mathrm{~h}$ later. During this verification test, subjects provided a written answer to each item. For each question, they had to type in the answer if they knew it; regardless of what they typed, they were then presented with two choices (one of which was always correct) to assess whether they could recognize the correct answer when it was presented. Subjects had to press one of two keys to indicate their choice of the correct answer; they could also press a third key if they thought that neither of the two answers presented was the correct one.

An additional episodic memory recognition test was administered prior to the verification test, in which subjects were presented with a set of questions, half of which were old, and were asked to indicate for each item whether it had been presented in the previous day's task. In this test, only questionparts (e.g., "Rome", meaning: "were you asked anything about Rome?") were presented, as opposed to the verification test in which subjects saw the whole question again (e.g., "Rome + river") and had to choose between two possible answers (e.g., "Po" or "Tiber"). Thus, there was no real overlap between target information in the episodic test and the verification test. Results from this episodic test strongly overlapped with the results of the verification test, however. Specifically, items given a ' $\mathrm{K}$ ' response in the cued retrieval task were also those that were episodically remembered best, followed by 'TOT' items, and then 'FOK' and 'DK' items. No meaningful episodic analysis of the imaging data could therefore be conducted and consequently this test will not be discussed further. 


\section{Functional imaging}

A 3T Siemens Allegra system was used to acquire highresolution T1-weighted anatomical images (MP-RAGE), and T2*weighted gradient-echo echo-planar functional images $(\mathrm{TR}=2000$ $\mathrm{ms}, \mathrm{TE}=40 \mathrm{~ms}, 21$ axial slices aligned parallel to the AC-PC plane, $5 \mathrm{~mm}$ thickness, $1 \mathrm{~mm}$ inter-slice skip, $200 \mathrm{~mm}$ FOV, $64 \times$ 64 matrix, 420 volume acquisitions per run). Four additional volumes were collected and discarded at the beginning of each run to allow for $\mathrm{T} 1$ equilibration.

\section{Preprocessing and data analysis}

Data were preprocessed using SPM99 (Wellcome Department of Cognitive Neurology, London). Images were first corrected for differences in slice acquisition timing by resampling all slices in time to match the first slice, followed by motion correction across both runs (using sinc interpolation). Data were then spatially normalized to an EPI template based upon the MNI305 stereotactic space (Cocosco et al., 1997). Images were resampled into 3-mm cubic voxels and then spatially smoothed with an 8-mm FWHM isotropic Gaussian kernel.

Statistical analysis was performed using the general linear model in SPM99.

Trials were sorted into bins depending on subjects' recall and recognition responses. Specifically, each trial was placed in one of four bins depending on the subject's response at the recall stage $(\mathrm{K}$, TOT, FOK, DK). These bins were then divided between items that were correctly answered at the recognition test stage (correct-K, correct-TOT, correct-FOK, correct-DK) or incorrectly answered (incorrect-K, incorrect-TOT, incorrect-FOK, incorrect-DK). There were too few trials categorized as incorrect-K, incorrect-TOT or incorrect-FOK to permit meaningful analysis $(N<15$; in no participant was there more than 15 items in each of these bins, with the means being 8 incorrect-K, 4 incorrect-TOT and 12 incorrectFOK). The remaining correct-K, correct-TOT, correct-FOK, correct-DK, and incorrect-DK trials were modeled using a canonical hemodynamic response. These effects were estimated using a subject-specific fixed-effects model, with session-specific effects and low-frequency signal components treated as confounds. Linear contrasts were used to obtain subject-specific estimates for each of the effects of interest. These estimates were entered into a secondlevel analysis treating subjects as a random effect, using a onesample $t$ test against a contrast value of 0 at each voxel. Statistical parametric maps were created for each contrast of interest, and were subsequently characterized using, at the voxel level, an uncorrected height threshold of $P<0.001$ and, at the cluster level, an extent threshold of $P<0.05$, corrected for the entire imaged volume. To further explore the nature of activation associated with each retrieval outcome condition, regions of interest (ROIs) were

Table 1

Retrieval outcome

\begin{tabular}{lrlll}
\hline & Don't know & $\begin{array}{l}\text { Feeling of } \\
\text { knowing }\end{array}$ & $\begin{array}{l}\text { Tip of the } \\
\text { tongue }\end{array}$ & Know \\
\hline Recall & $120(0.36)$ & $53(0.16)$ & $31(0.10)$ & $127(0.38)$ \\
Verified & $49(0.15)$ & $40(0.12)$ & $27(0.08)$ & $119(0.36)$ \\
\hline
\end{tabular}

Distribution of retrieval outcome responses. Recall row: Mean (prop.) of response type at the recall stage. Verified row: Mean (prop.) of responses that were verified at the recognition stage.
Table 2

Proportion of each response type in the present and previous study

\begin{tabular}{lllll}
\hline & Don't know & $\begin{array}{l}\text { Feeling of } \\
\text { knowing }\end{array}$ & $\begin{array}{l}\text { Tip of the } \\
\text { tongue }\end{array}$ & Know \\
\hline Present study & 0.36 & 0.16 & 0.10 & 0.38 \\
Maril et al. (2001) & 0.51 & - & 0.9 & 0.40 \\
\hline
\end{tabular}

Comparing the distributions of retrieval outcome responses between Maril et al. (2001), in which three response options were available (K, TOT, and DK) with the same distribution in the present study, which has an additional FOK response option.

identified from clusters that survived the thresholding criteria. The hemodynamic responses were extracted from each ROI on a subject-by-subject basis, and were subjected to subsequent repeated measures analyses that included factors for condition (correct-K, correct-TOT, correct-FOK, correct-DK, incorrect-DK) and peri-stimulus time ( $2-6 \mathrm{~s})$; the level of significance for ROIs was $P<0.001$.

Since the reaction times associated with TOT and FOK responses were significantly longer than those associated with $\mathrm{K}$ and DK responses (see Results), an additional analysis was performed, where reaction time (RT) was entered into the analysis as a parametric modulator.

\section{Results}

\section{Behavioral data}

Subjects were scanned while responding to each of 336 general knowledge questions. Depending on each subject's responses, trials were sorted into four response-based bins: Know (K), Don't Know (DK) Feeling-of-Knowing (FOK), and Tip-of-the-Tongue (TOT). The distribution of responses among these bins is detailed in the first row of Table 1. A TOT response was given in about $10 \%$ of all trials (consistent with findings in the behavioral TOT literature and in our previous fMRI study (Maril et al., 2001). A FOK response was given for $16 \%$ of all trials, and the remaining responses were divided fairly evenly between the Know and Don't Know bins (no statistically significant difference, $P=0.42$; all other differences were significant at $P<0.001$ ).

Comparing the response distributions of the present study with that of Maril et al. (2001), in which only three response options were available (K, TOT, and DK), it seems likely that retrieval outcomes that were given the FOK response option in the present study might have previously resulted in a DK response in the Maril et al. (2001) study: the proportion of K and TOT trials is very similar in the two studies, whereas fewer trials in the present study were given a DK response (see Table 2).

On a post-scanning verification test (see Materials and methods), almost all $\mathrm{K}$ responses were correctly recognized, as were the majority of the TOT and FOK trials; performance on the recognition task for DK trials was at chance (See second row of Table 2).

Mean (standard error) median reaction times during the scanned task differed across conditions [K: 1942 (126); DK: 1956 (130); FOK: 2569 (78); TOT: $2764(63) \mathrm{msec}, F(3,42)=40.9, P<$ $0.0001]$. Planned contrasts revealed that while the RT difference between $\mathrm{K}$ and $\mathrm{DK}$ trials was not significant $(t(14)=0.013)$, all other retrieval conditions differed significantly from each other (TOT and FOK: $t(14)=3.2 . P<0.005$; TOT and $\mathrm{K}: t(14)=8.6$, 
$P<0.001$; FOK and $\mathrm{K}: t(14)=6.7, P<0.001$; TOT and DK: $t(14)=7.7, P<0.001$; FOK and DK: $t(14)=6.7, P<0.001)$.

\section{Imaging data}

The fMRI data were analyzed to assess activation associated with performance of the semantic retrieval task to ensure general replication of the results from the original TOT study, as well as other semantic retrieval experiments. Performance of the semantic retrieval task, as reflected in the comparison of all retrieval conditions vs. baseline, elicited activation in numerous brain regions, including regions in left dorsolateral prefrontal, bilateral inferior frontal, bilateral parietal, and bilateral occipital cortices (Fig. 1). These regions generally converge with prior neuroimaging studies of semantic retrieval (e.g., Gabrieli et al., 1996; Petersen et al., 1988; Wagner et al., 1998; for review, see Cabeza and Nyberg, 2000). Next, regions that exhibited significantly greater activation for TOT over $\mathrm{K}, \mathrm{FOK}$, and DK were identified using the task $>$ fixation contrast and direct contrasts between TOT and the other retrieval types. These comparisons revealed significant TOT-related activation (i.e., TOT $>$ all other retrieval conditions) in the same regions as were observed previously: ACC, right DLPFC, and RIFC (Fig. 2a-c). In addition, significant TOT-related activation was observed in bilateral anterior frontal cortex (Fig. 2d; Table 3, top).

There were also regions in which both TOT and FOK responses elicited comparable levels of activation, which was significantly greater than the levels of activation that were associated with the other response types: posterior medial parietal cortex (Fig. 3a), bilateral lateral parietal cortex (Fig. 3b), and bilateral superior PFC (pre-SMA, BA6/4). Thus, unlike the ACC, right DLPFC, RIFC, and anterior $\mathrm{PFC}$, activation in these regions was not unique to TOT (Table 3, middle).

The RT analysis revealed no areas in frontal cortex in which activation was correlated with time on task. Two regions in the medial and left parietal lobes were identified in this analysis, whose coordinates were somewhat close to those of the regions whose activation was found in the initial analysis to be modulated by both TOT and FOK.

Using the same task $>$ baseline contrast, $\mathrm{K}$ responses were found to elicit activation in left anterior hippocampus (Fig. 4) which was significantly greater than all other retrieval types, with no additional differences among them (Table 3, bottom).

No region was found in which FOK or DK elicited the highest activation, compared to the other retrieval types.

\section{Discussion}

The objective of the present study was to examine whether the pattern of TOT-related activation observed in a previous study (Maril et al., 2001) was indeed specific to the TOT, or whether other intermediate recall states would produce a similar pattern of activation. In the previous study, TOT-related activations observed in ACC, right DLPFC and right inferior frontal cortex were interpreted in terms of cognitive processes that specifically accompany the TOT state, such as retrieval and evaluation of partial information (see below). However, it is possible that some of these activations reflect a more general 'metacognitive' process, such as evaluating one's level of knowledge on a subject, evaluating one's probability of getting the correct answer in time, deciding on a response, etc. If the activations observed during a TOT state reflect metacognitive processes, then other processes that place similar demands on the metacognitive system could be expected to produce a similar pattern of activations. To test this question, the present study directly contrasted feeling of knowing to the TOT state within subjects and within memory systems.

The present study replicated and extended the characterization of TOT-related (TOT $>$ all other retrieval types) activation, to include anterior cingulate, right dorsal PFC, right inferior PFC, and bilateral anterior PFC. Both TOT and FOK were associated with high levels of activations in lateral and posterior medial parietal regions. However, there was no significant difference between TOT- and FOK-related activation in these regions: both intermediate recall states elicited a high level of activation compared to Know and Don't Know recall responses.

Consistent with the results of our previous TOT imaging study (Maril et al., 2001), the ACC, right DLPFC, and RIFC regions were observed to be specifically engaged by the TOT state, as was anterior PFC bilaterally. As discussed in the original study, activation in these regions would be expected in light of the phenomenology of TOT, specifically the recovery of partial information about the target and target-related items. The ACC and prefrontal cortex have been posited as two components of a cognitive control system, where the ACC has been hypothesized to contribute through conflict monitoring and subsequent facilitation of PFC function (Cohen et al., 2000; MacDonald et al., 2000); activation in the right DLPFC (Henson et al., 1999a,b) and anterior PFC (Allan et al., 2001; Rugg et al., 1998) have been suggested to be modulated by demands on post-retrieval monitoring operations. While in a TOT state, the recovered partial information (Brown and McNeill, 1966; Schwartz, 1999) places demands on this system:
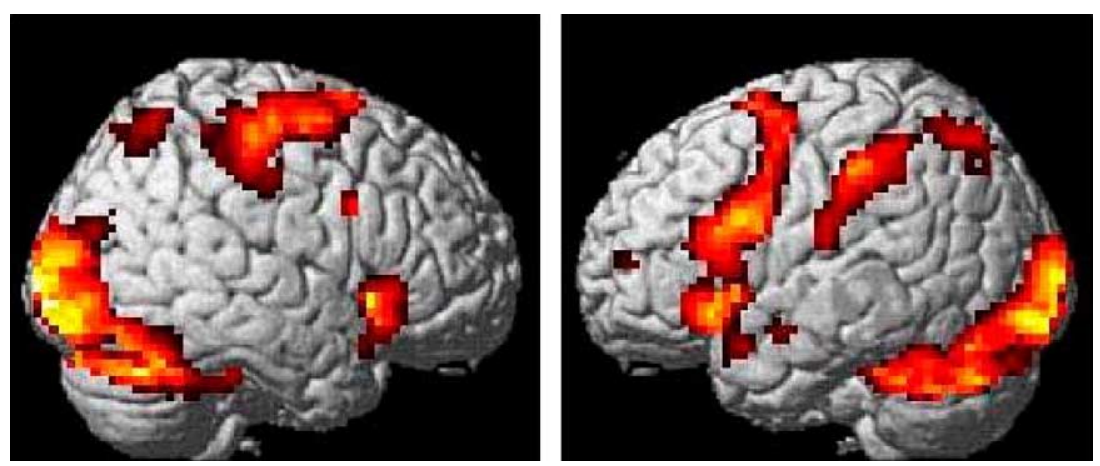

Fig. 1. Results of the comparison of all retrieval conditions to baseline (semantic retrieval task). The activation maps are superimposed onto the left and right hemispheres of a canonical brain. 

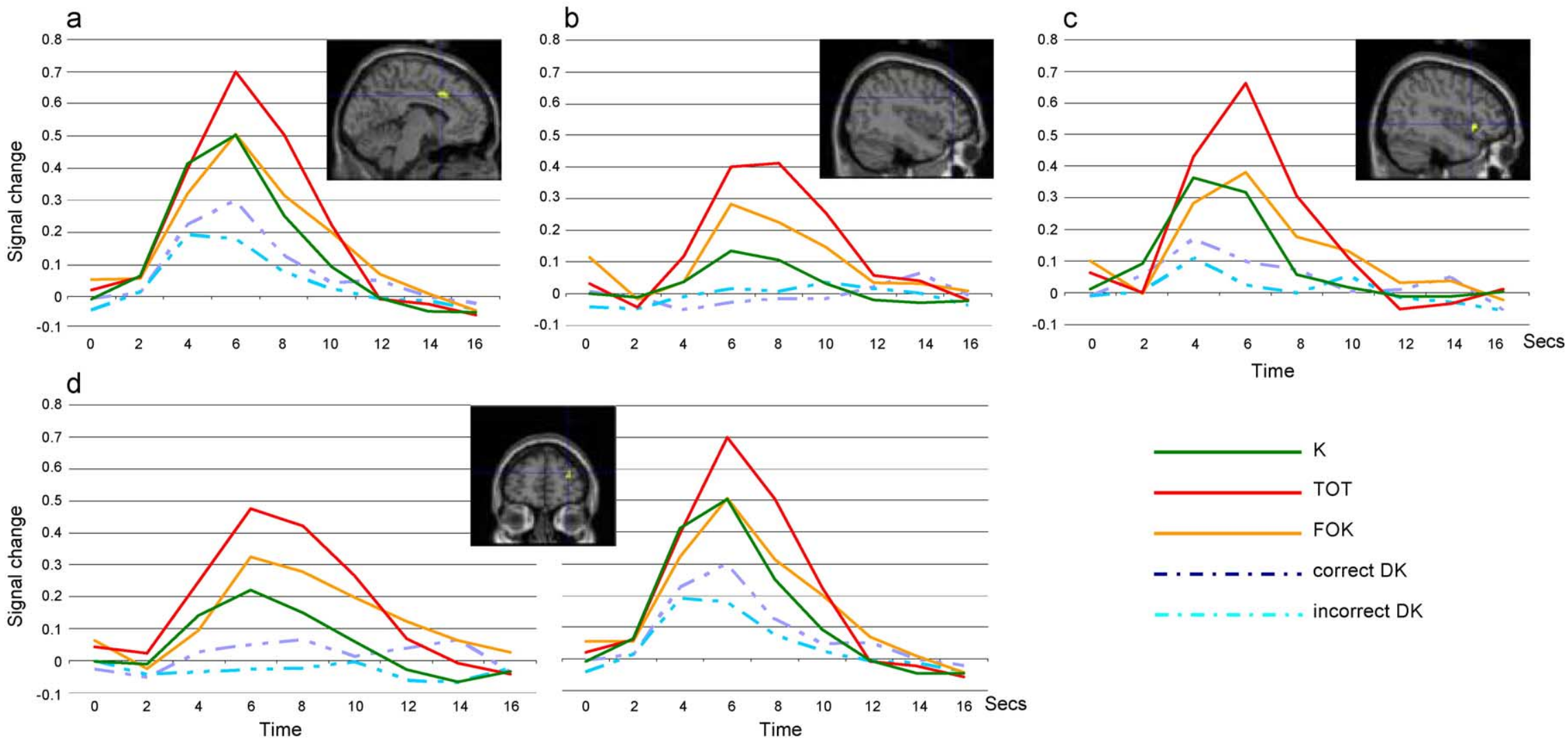

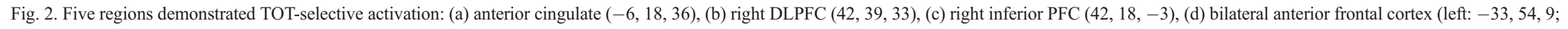
right: 30,54, 21). Displayed are sections through each region, and averaged event-related responses associated with each retrieval outcome. Coordinates are in MNI space. 

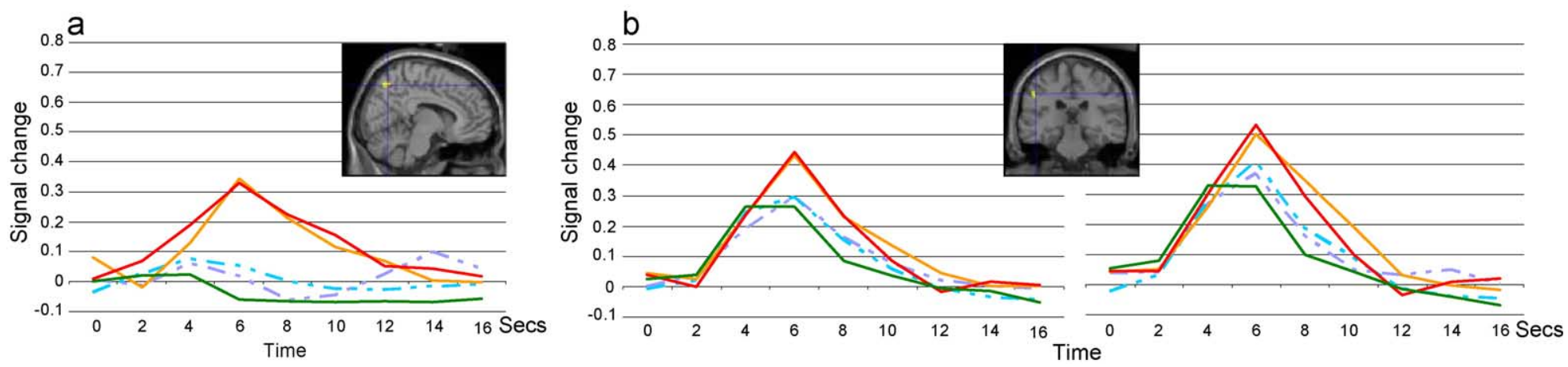

Fig. 3. Similar activations for TOT and FOK, which where greater than that associated with the other retrieval outcomes were observed in medial (a. 9, $-66,51)$ and lateral (b. left: $-48,-27,39$; right: $48,-27,48$ ) parietal regions. Displayed are functionally defined ROIs and the resultant averaged event-related responses. Coordinates are in MNI space. 
Table 3

Cortical and medial temporal regions modulated by response type

\begin{tabular}{lrrrrr}
\hline Region & $X$ & $Y$ & $Z$ & Max T & \# Voxels \\
\hline TOT $>$ all else & & & & & \\
Anterior cingulate cortex & -6 & 18 & 36 & 8.81 & 70 \\
Right dorsolateral PFC & 42 & 36 & 33 & 6.20 & 59 \\
Right inferior PFC & 42 & 18 & -3 & 7.98 & 53 \\
Left anterior PFC & -33 & 54 & 15 & 8.07 & 63 \\
Right anterior PFC & 30 & 51 & 18 & 6.70 & 75
\end{tabular}

TOT $=$ FOK $>$ all else

Medial parietal cortex

Left lateral parietal cortex

$\begin{array}{rrrrr}9 & -69 & 54 & 5.87 & 54 \\ -48 & -27 & 39 & 10.90 & 63 \\ 48 & -27 & 48 & 10.00 & 60\end{array}$

$K>$ all else

$\begin{array}{llllll}\text { Left anterior hippocampus } & -30 & -18 & -18 & 5.73 & 34\end{array}$

Regions of interest for the different activation patterns. Listed are the peak voxels for each region. Coordinates are in MNI space.

some information may be facilitative to the search process, and will need to be evaluated as such and utilized in convergence toward the answer; some information may be detrimental to the attempt to recover target knowledge, such as competing items that resemble the target word either phonologically or semantically (e.g., Reason and Lucas, 1984). Thus, the recovered partial information may lead to a strong sense of familiarity about the non-retrieved target, but may give rise to conflict or competition during attempts to resolve the TOT, thereby engaging the brain systems required to detect and manage cognitive conflict, as well as regions required to evaluate the internally generated products of retrieval.

The phenomenological experience that an item is known, coupled with the surprising inability to retrieve it that is typical of the TOT state, suggests a possible interpretation of the TOT-related activation observed in the frontopolar region. Theories of anterior PFC (BA 10) suggest that it plays a specific executive role in memory and attention, such as evaluating and managing one's own thought processes (Burgess et al., in press; Simons et al., submitted for publication). For example, anterior frontal regions become activated when a task requires integration and processing of multiple relations simultaneously (Christoff et al., 2001) or holding in mind goals while allocating attention to secondary goals (Braver and Bongiolatti, 2002; Koechlin et al., 1999). Similarly, anterior PFC activation has been observed during prospective memory tasks that involve maintaining an intention while performing an ongoing task (Burgess et al., 2001, 2003). In this view, anterior PFC may interact with the ACC-right DLPFC circuit in managing the relatively complicated retrieval process in a TOT state. The region might support processes involved in switching between evaluation of retrieval products and further search; holding in mind the information about the task and the criteria for a TOT response while the retrieval process itself is carried out; or perhaps actively biasing attention away from the phonologically or semantically similar competing items that obstruct retrieval of the target (Burgess et al., in press).

Another region that exhibited TOT-related activation was the right inferior frontal cortex, replicating a similar observation by Maril et al. (2001). Maril et al. speculated, on the basis of informal observation, that subjects adopted a strategy that relies heavily on visual-spatial information when trying to resolve TOT, for example, trying to 'read' the name of an author from the imagined cover of a book (for visual-spatial modulation of right inferior PFC activation see Awh and Jonides, 1998; Wagner, 1999; but see Fletcher et al. (2002) for a more comprehensive view of PFC laterality). Alternatively, this region has been implicated in finegrained phonological processing of words, at the level of phonemes or single syllables (Poldrack et al., 1999; Pugh et al., 1997), as opposed to a similar region in the left hemisphere in which phonological processing occurs for larger units, such as whole words. While retrieval of phonetic information occurs also for $\mathrm{K}$ and FOK trials, the nature of such retrieved information may be different: $\mathrm{K}$ trials are likely to be accompanied by a retrieval of the whole word, and FOK retrieval attempts may elicit poor partial phonetic information relative to TOT trials, as suggested by robust behavioral findings about partial phonological information retrieved while in a TOT state (for review of evidence, see Brown, 1991). It is difficult to distinguish between these two possible explanations on the basis of the present data.

A recent fMRI study of semantic retrieval was conducted by Kikyo et al. (2002), in which subjects were presented with general knowledge questions in the scanner, and had to reply Yes/No to successful recall of the answer. In a post-scanning test, they had to rate their feeling of knowing for each unrecalled item, from 1 (did not know) to 3 (could definitely recall given more time/hints), with an intermediate level of 2 defined as "I probably would recognize" (p. 177). Kikyo et al. performed a parametric analysis using the FOK ratings as the regressor of interest, and found activation in regions in bilateral IFG, LMFG, and ACC to be parametrically modulated by the FOK rating. They also report that among these regions, bilateral IFG was not activated during successful recall trials "suggesting their particular role in metamemory processing" (p.182). Though seemingly similar, comparing the present experiment's findings to the results reported by Kikyo et al. is complicated by factors at both the cognitive and the neuroimaging levels. First, Kikyo et al. set the parametric modulator to be smallest in DK trials and highest in strong FOK trials, with $\mathrm{K}$ trials not included. It is not clear what is the theoretical basis for thinking of FOK as a variant of DK but not as a variant of $\mathrm{K}$ recall states. A parametric analysis performed in the present experiment, rating DK as 1 , FOK as 2, TOT as 3 and $\mathrm{K}$ as 4 , did not yield any region in

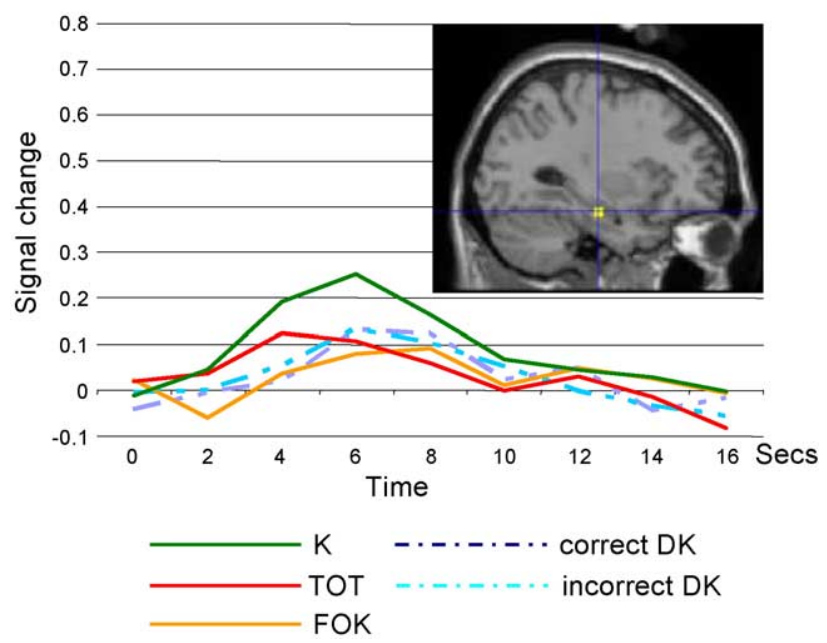

Fig. 4. Successful recall elicited greater activation than all other retrieval responses in left anterior hippocampus $(-30,-18,-18)$. Displayed is a sagittal section through the functionally defined ROI. Coordinates are in MNI space. 
the prefrontal cortex whose activation was linearly modulated by that regressor, suggesting that subjects' responses reflected qualitatively different retrieval states as opposed to a simple quantitative heuristic such as response confidence, for example. Second, extensive piloting guided us to instruct subjects to respond with the K option only after being able to silently 'say the answer'. When not so instructed, TOT experiences may go unnoticed: subjects may feel very confident that they know the answer, but only when they try to actually produce it do they notice their inability to do so. It is thus possible that the K bin in Kikyo et al.'s experiment includes both K and TOT trials. Third, Kikyo et al. did not use a baseline task. Thus, for example, they report that bilateral IFG regions are not recruited in successful recall, while in the present study, we find a remarkably similar region in LIFG to be activated by K, TOT and FOK (Kikyo et al.: $-30,28,-10$; present experiment: $-35,23,-7$, coordinates in Talairach space). These conflicting findings may be due solely to the different use of baseline.

Both TOT and FOK activated regions in medial and lateral parietal cortex to a similar extent, and significantly more than $\mathrm{K}$ and DK trials. Lateral parietal regions have been suggested to be involved in working memory, holding a representation on line (Jonides et al., 1998). The increased activation in these regions during both TOT and FOK may reflect the partial information held in working memory while a response decision is being made, on the basis of this incomplete set of related items. K and DK trials would not be expected to similarly activate these regions since they are made relatively quickly on the basis of a full recovery of one item of information that does not require further processing $(\mathrm{K})$ or on the basis on the absence of the sought-for information (DK). To the extent that both TOT and FOK trials are accompanied by partial information to be processed (to stimulate and facilitate further search, to be evaluated, etc.), it may be surprising that activation in the ACC, right DLPFC and the frontopolar regions is modulated mainly by TOT but not by FOK trials. One possible explanation, albeit speculative, is that TOT and FOK differ in the richness of the content of retrieved partial information; if FOK is accompanied by one or two reoccurring items held on line, and TOT is accompanied by a variety of related items to be held in short-term memory, the FOK state does not provide a rich input for anterior processing, while the TOT state does. This idea merits examination in future behavioral and imaging studies.

The posterior medial parietal region observed here has been implicated in connection to visual imagery (Fletcher et al., 1995; Knauff et al., 2002, but see Buckner et al., 1996). A possible interpretation for the TOT- and FOK-induced activation in this region may be that both states stimulate the use of visual imagery in the attempt to resolve them. That subjects use mental imagery in trying to resolve a TOT is supported by subjects' informal reports about using this strategy. Again, that imagery is used when FOK is experienced, while possible, is highly speculative at this point. An alternative, more conservative possible interpretation of the parietal activation observed for TOT and FOK trials, is connected to their frequency compared to K and DK trials. As mentioned in Results, $\mathrm{K}$ and DK trials constitute the vast majority of the trials, with TOT and FOK together accounting for less than $30 \%$ of all trials. As medial and lateral parietal regions have been suggested to be part of a circuit involved in attending to and planning motor responses (Johnson et al., 2002), especially non-habitual ones (Le et al., 1998; Sylvester et al., 2003), the experimental design, which did not include other types of low frequency responses to control for this factor, does not permit exclusion of this interpretation.

There were significantly more FOK responses than TOT responses (see Table 1), but no brain region showed a pattern of activation that reflected this behavioral difference. Instead, FOK was observed to be associated with levels of activation that were either equal to or lower than the levels of activation associated with TOT.

Though not central to the main hypotheses in this study, it is worth noting that $\mathrm{K}$ trials activated a region in the anterior hippocampus more than all other retrieval types. This observation is somewhat surprising since the task at hand was retrieval from semantic memory while this region is strongly connected to episodic memory. It could be the case that this activation reflects the episodic encoding of the $\mathrm{K}$ trials. It is similarly possible that this activation reflects the re-encoding of the retrieved information; however, it is not clear why information retrieved in the $\mathrm{K}$ trials would be re-encoded while information retrieved in the TOT trials, for example, would not.

In summary, the present experiment replicated and extended findings from a previous semantic retrieval experiment (Maril et al., 2001). Activation in ACC, right DLPFC, RIFC, and anterior PFC was unique to TOT trials; medial and lateral parietal regions were activated by both TOT and FOK, that is, activations in these regions were not exclusively TOT-related. Moreover, directly comparing TOT and FOK, the results of this study suggest that activations in frontal areas reflect processes that are specifically associated with TOT, rather than more general metacognitive processes. In the behavioral literature, TOT and FOK are usually regarded as two levels of an essentially unitary process, with TOT being thought of as a stronger instance of FOK. As no dissociation was observed in the present study, the results are inconclusive with regard to the type of difference between TOT and FOK, that is, there is a quantitative or a qualitative difference between them. However, with TOT but not FOK eliciting activations in frontal areas, it may be the case that TOT is a cognitive state that is not merely a strong instance of FOK, but rather that there may exist a qualitative difference between the two states. This issue needs to be critically tested in future research.

\section{Acknowledgments}

We are grateful to Russ Poldrack and Jason Mitchell for development of analysis tools. This work was supported by NIH Grant MH60941. JSS was additionally supported by Wellcome Trust Grant 061171.

\section{References}

Allan, K., Wolf, H.A., Rosenthal, C.R., Rugg, M.D., 2001. The effect of retrieval cues on post-retrieval monitoring in episodic memory: an electrophysiological study. Cogn. Brain Res. 12, 289-299.

Awh, E., Jonides, J., 1998. Spatial working memory and spatial selective attention. In: Parasuraman, R. (Ed.), The Attentive Brain. MIT Press, Cambridge, MA, USA, pp. 353-380.

Braver, T.S., Bongiolatti, S.R., 2002. The role of frontopolar cortex in subgoal processing during working memory. NeuroImage 15, 523-536.

Brown, A.S., 1991. A review of the tip-of-the-tongue experience. Psychol. Bull. 109, 204-223.

Brown, R., McNeill, D., 1966. The "tip of the tongue" phenomenon. J. Verbal Learn. Verbal Behav. 5, 325-337.

Buckner, R.L., Raichle, M.E., Miezin, F.M., Petersen, S.E., 1996. Func- 
tional anatomic studies of memory retrieval for auditory words and visual pictures. J. Neurosci. 16, 6219-6235.

Burgess, P.W., Quayle, A., Frith, C.D., 2001. Brain regions involved in prospective memory as determined by positron emission tomography. Neuropsychologia 39, 545-555.

Burgess, P.W., Scott, S.K., Frith, C.D., 2003. The role of the rostral frontal cortex (area 10) in prospective memory: A lateral versus medial dissociation. Neuropsychologia 41, 906-918.

Burgess, P.W., Simons, J.S., Dumontheil, I., Gilbert, S.J. (in press). The gateway hypothesis of rostral PFC function. In: Duncan, J., Phillips, L., McLeod, P. (Eds.), Speed, Control and Ageing: In Honour of Patrick Rabbitt. Oxford Univ. Press, Oxford.

Cabeza, R., Nyberg, L., 2000. Imaging cognition II: an empirical review of 275 PET and fMRI studies. J. Cogn. Neurosci. 12, 1-47.

Christoff, K., Prabhakaran, V., Dorfman, J., Zhao, Z., Kroger, J.K., Holyoak, K.J., Gabrieli, J.D.E., 2001. Rostrolateral prefrontal cortex involvement in relational integration during reasoning. Neurolmage 14, $1136-1149$

Cocosco, C.A., Kollokian, V., Kwan, R.K.S., Evans, A.C., 1997. Brainweb: online interface to a 3D MRI simulated brain database. NeuroImage 5, 425.

Cohen, G., Faulkner, D., 1986. Memory for proper names: age differences in retrieval. Br. J. Dev. Psychol. 4, 187-197.

Cohen, J.D., Botvinik, M., Carter, C.S., 2000. Anterior cingulate and prefrontal cortex: who's in control? Nat. Neurosci. 3, 421-423.

Dale, A.M., 1999. Optimal experimental design for event-related fMRI. Hum. Brain Mapp. 8, 109-114.

Fletcher, P.C., Frith, C.D., Baker, S.C., Shallice, T., Frackowiak, R.S.J., Dolan, R.J., 1995. The mind's eye-precuneus activation in memory related imagery. NeuroReport 2, 195-200.

Fletcher, P.C., Palomero-Gallagher, N., Zafiris, O., Fink, G.R., Tyler, L.K., Zilles, K., 2002. The influence of explicit instructions and stimulus material on lateral frontal responses to an encoding task. NeuroImage 17, 780-791

Gabrieli, J.D.E., Desmond, J.E., Demb, J.B., Wagner, A.D., Stone, M.V., Vaydia, C.J., Glover, G.H., 1996. Functional magnetic resonance imaging of semantic memory processes in the frontal lobes. Psychol. Sci. 7, 278-283.

Hart, J.T., 1965. Memory and the feeling-of-knowing experience. J. Educ. Psychol. 56, 208-216.

Henson, R.N., Shallice, T., Dolan, R.J., 1999. Right prefrontal cortex and episodic memory retrieval: a functional MRI test of the monitoring hypothesis. Brain 122, 1367-1381.

Henson, R.N.A., Rugg, M.D., Shallice, T., Josephs, O., Dolan, R.J., 1999. Recollection and familiarity in recognition memory: an eventrelated functional magnetic resonance imaging study. J. Neurosci. 19, $3962-3972$.

Johnson, S.H., Rotte, M., Grafton, S.T., Hinrichs, H., Gazzaniga, M.S., Heinze, H.J., 2002. Selective activation of a parietofrontal circuit during implicitly imagined prehension. NeuroImage 17, 1693-1704.

Jonides, J., Schumacher, E.H., Smith, E.E., Koeppe, R.A., Awh, E., ReuterLorenz, P.A., Marshuetz, C., Willis, C.R., 1998. The role of parietal cortex in verbal working memory. J. Neurosci. 18, 5026-5034.

Kikyo, H., Ohki, K., Miyashita, Y., 2002. Neural correlates for feeling-ofknowing: an fMRI parametric analysis. Neuron 36, 177-186.

Knauff, M., Mulack, T., Kassubek, J., Salih, H.R., Greenlee, M.W., 2002. Spatial imagery in deductive reasoning: a functional MRI study. Brain Res. Cogn. Brain Res. 13, 203-212.

Koechlin, E., Basso, G., Pietrini, P., Panzer, S., Grafman, J., 1999. The role of the anterior prefrontal cortex in human cognition. Nature 399, 148-151.
Koriat, A., Leiblich, I., 1974. What does a person in a "tot" know that a person in a "don't know" state doesn't know. Mem. Cogn. 2, 647-655.

Le, T.H., Pardo, J.V., Hu, X., 1998. 4 T-fMRI study of nonspatial shifting of selective attention: cerebellar and parietal contributions. J. Neurophysiol. 79, 1535-1548.

MacDonald, A.W., Cohen, J.D., Stenger, V.A., Carter, C.S., 2000 Dissociating the role of the dorsolateral prefrontal and anterior cingulate cortex in cognitive control. Science 288, 1835-1838.

Maril, A., Wagner, A.D., Schacter, D.L., 2001. On the tip of the tongue: an event-related fMRI study of semantic retrieval failure and cognitive conflict. Neuron 31, 653-660.

Maril, A., Simons, J.S., Mitchell, J.P., Schwartz, B.L., Schacter, DL., 2003. Feeling-of-knowing in episodic memory: an event-related fMRI study. NeuroImage 18 (4), 827-836.

Miozzo, M., Caramazza, A., 1997. Retrieval of lexical-syntactic features in tip-of-the-tongue state. J. Exp. Psychol., Learn. Mem. Cogn. 23, $1410-1423$.

Petersen, S.E., Fox, P.T., Posner, M.I., Mintum, M., Raichle, M.E., 1988. Positron emission tomographic studies of the cortical anatomy of single-word processing. Nature 331, 585-589.

Poldrack, R.A., Wagner, A.D., Prull, M.W., Desmond, J.E., Glover, G.H., Gabrieli, J.D.E., 1999. Functional specialization for semantic and phonological processing in the left inferior prefrontal cortex. NeuroImage 10, 15-35.

Pugh, K.R., Shaywitz, B.A., Shaywitz, S.E., Shankweiler, D.P., Katz, L., Fletcher, J.M., Skudlarski, P., Fulbright, R.K., Constable, R.T., Bronen, R.A., et al., 1997. Predicting reading performance from neuroimaging profiles: the cerebral basis of phonological effects in printed word identification. J. Exp. Psychol., Hum. Percept. Perform. 23, 299-318.

Reason, J.T., Lucas, D., 1984. Using cognitive diaries to investigate naturally occurring memory blocks. In: Harris, J.E., Morris, P.E. (Eds.), Everyday Memory, Actions and Absentmindedness. Academic Press, Orlando, FL, pp. 53-69.

Rubin, D.C., 1975. Within word structure in the tip-of-the-tongue phenomenon. J. Verbal Learn. Verbal Behav. 14, 392-397.

Rugg, M.D., Fletcher, P.C., Allan, A., Frith, C.D., Frackowiak, R.S.J., Dolan, R.J., 1998. Neural correlates of memory retrieval during recognition memory and cued recall. NeuroImage 8, 262-273.

Schwartz, B.L., 1999. Sparkling at the end of the tongue: the etiology of tip-of-the-tongue phenomenology. Psychon. Bull. Rev. 6, 379-393.

Schwartz, B.L., 2002. Tip-of-the-Tongue States: Phenomenology, Mechanism, and Lexical Retrieval. Erlbaum, Mahwah, NJ.

Simons, J.S., Owen, A.M., Fletcher, P.C., Burgess, P.W., submitted for publication. Anterior prefrontal cortex and the recollection of internallygenerated thoughts.

Sylvester, C.Y., Wager, T.D., Lacey, S.C., Hernandez, L., Nichols, T.E., Smith, E.E., Jonides, J., 2003. Switching attention and resolving interference: fMRI measures of executive functions. Neuropsychologia $41,357-370$.

Vigliocco, G., Vinson, D.P., Martin, R.C., Garrett, M.F., 1999. Is "count" and "mass" information available when the noun is not? An investigation of tip of the tongue states and anomia. J. Mem. Lang. 40, 534-558.

Wagner, A.D., 1999. Working memory contributions to human learning and remembering. Neuron 22, 19-22.

Wagner, A.D., Schacter, D.L., Rotte, M., Koutstaal, W., Maril, A., Dale, A.M., Rosen, B.R., Buckner, R.L., 1998. Building memories: remembering and forgetting of verbal experiences as predicted by brain activity. Science $281,1188-1191$ 